\title{
SOLUTION OF IRVING'S RAMSEY PROBLEM
}

\author{
by HEIKO HARBORTH and HEINZ-MICHAEL NITZSCHKE
}

(Received 6 June, 1979)

In [1] the following question was posed by R. W. Irving (see also Conjecture 4.10 in [4]): Is there an edge 2-colouring of the complete bipartite graph $K_{13,17}$ with no monochromatic $K_{3,3}$ ? We give a negative answer in this note (Theorem 2). Furthermore we prove Conjecture 4.11 (i) of [4] (Theorem 1), that is, any edge 2-coloured $K_{2 n+1,4 n-3}$ contains a monochromatic $K_{2, n}$ with the 2 and $n$ vertices a subset of the $2 n+1$ and $4 n-3$ vertices, respectively. Theorem 1 is a consequence of Satz 4 in [3], however, we give a direct proof here.

Instead of edge coloured complete bipartite graphs $K_{x, y}$ we use $0-1$-matrices $M=$ $\left(m_{i, j}\right)$, where $m_{i, j}=0$ or $1(1 \leqq i \leqq x, 1 \leqq j \leqq y)$, if the edge $(i, j)$ of $K_{x, y}$ is of the first or second colour, respectively.

LEMMA 1. If $p_{i j}, p_{i k}$, and $p_{j k}$ denote the numbers of equal columns (both entries 0 or both entries 1) in the three pairs of rows of any triple of rows $(i, j, k)$ in any 0-1-matrix with $c$ columns, then

$$
p_{i j}+p_{i k}+p_{j k} \equiv c(\bmod 2) \text {. }
$$

Proof. Each column contributes 1 or 3 to the sum on the left-hand side.

In the following we denote by $[x]$ and $\{x\}$ the greatest integer $\leqq x$ and the smallest integer $\geqq x$, respectively.

Theorem 1. Any $(2 n+1,4 n-3)$-0-1-matrix contains a $(2, n)$-submatrix with entries 0 only, or 1 only.

Proof. Any column of a $(2 n+1,4 n-3)-0-1-$ matrix $M$ contains at least $\left(\begin{array}{l}n \\ 2\end{array}\right)+$ $\left(\begin{array}{c}n+1 \\ 2\end{array}\right)$ pairs of equal entries. Thus for the total number $A$ of equal pairs in all columns of $M$ we obtain

$$
A \geqq(4 n-3)\left(\left(\begin{array}{c}
n \\
2
\end{array}\right)+\left(\begin{array}{c}
n+1 \\
2
\end{array}\right)\right)=4 n^{3}-3 n^{2} .
$$

Using the pigeonhole principle there is at least one pair of rows in $M$ with

$$
\left.p=\left\{\begin{array}{c}
\frac{4 n^{3}-3 n^{2}}{(2 n+1} \\
2
\end{array}\right)\right\}=\left\{2 n-2-\frac{n-2}{2 n+1}\right\}=2 n-2
$$

equal columns for $n \geqq 2$ (if $n=1$, then Theorem 1 is trivial).

Glasgow Math. J. 21 (1980) 187-197. 
We assume that no pair of rows in $M$ has more than $p$ equal columns. In any triple of rows at most two pairs have $p$ equal columns (Lemma 1). Then the famous theorem of Turán ([2], p. 17) implies that at most $\left[(2 n+1)^{2} / 4\right]=n^{2}+n$ pairs of rows in $M$ have $p$ equal columns. It follows that

$$
A \leqq\left(n^{2}+n\right)(2 n-2)+\left(\left(\begin{array}{c}
2 n+1 \\
2
\end{array}\right)-\left(n^{2}+n\right)\right)(2 n-3)=4 n^{3}-3 n^{2}-2 n
$$

which contradicts (2). Thus at least one pair of rows in $M$ has $p+1=2 n-1$ equal columns, that means, $\left\{\frac{2 n-1}{2}\right\}=n$ columns have entries 0 only, or 1 only.

TheOREM 2. Any $(13,17)-0-1$-matrix contains a $(3,3)$-submatrix with entries 0 only, or 1 only.

Proof. We denote by $B=\left(b_{i, j}\right)$ a $(13,17)-0-1$-matrix, by $N$ the $(3,3)$-matrix with entries 0 only, and by $\bar{M}$ the matrix $M$ with 0 and 1 interchanged. If $M$ contains a submatrix $S$, we will write $S \subset M$. The proof is divided into the following Lemmas. Those parts of their proofs which follow by changing 0 and 1 are omitted.

We consider the matrices $S_{i}(1 \leqq i \leqq 10)$ shown opposite as submatrices of $B$ up to exchanges of rows or columns.

LEMMA 2. If $S_{1}$ or $\bar{S}_{1} \subset B$, then $N$ or $\bar{N} \subset B$,

Proof. If $S_{1} \subset B$, then either $N \subset B$, or every row of that $(11,6)$-submatrix $M$ of $B$ determined by the columns of $S_{1}$ contains at least 4 entries 1 , that is, $\left(\begin{array}{l}4 \\ 3\end{array}\right)=4$ triples of entries 1 . Then any distribution of these 44 triples among the 6 columns of $M$ guarantees $\bar{N} \subset M$, since $2\left(\begin{array}{l}6 \\ 3\end{array}\right)<44$.

LemMa 3. If $S_{2}, S_{2}^{T}, \bar{S}_{2}$ or $\bar{S}_{2}^{T} \subset B$, then $N$ or $\bar{N} \subset B$.

Proof. Let $S_{2} \subset B$, or $S_{2} \subset B^{T}$, which corresponds to $S_{2}^{T} \subset B$. The first 5 columns and the last 7 rows of $S_{2}$ determine $M \subset S_{2}$. Either $N \subset S_{2}$, or every row of $M$ contains at least 3 entries 1 , that is, $\left(\begin{array}{l}3 \\ 2\end{array}\right)=3$ pairs of entries 1 . In any distribution of these 21 pairs among the 5 columns of $M$ there are 2 columns with 3 pairs of entries 1 in a row, since $2\left(\begin{array}{l}5 \\ 2\end{array}\right)<21$. Together with column 6 of $S_{2}$, it follows that $\bar{N} \subset S_{2}$.

LEMMA 4. If $S_{3}$ or $\bar{S}_{3} \subset B$, then $N$ or $\bar{N} \subset B$.

Proof. If $S_{3}$ is in the first rows and columns of $B$, then rows 6 to 13 , and columns 3 to 17 determine a $(8,15)$-submatrix $M$ of $B$. If there is one row of $M$ with more than 6 entries 1 , then Lemma 3 can be used. Otherwise $M$ has at least $8 \times 9=72$ entries 0 . Let $s_{i}$ 

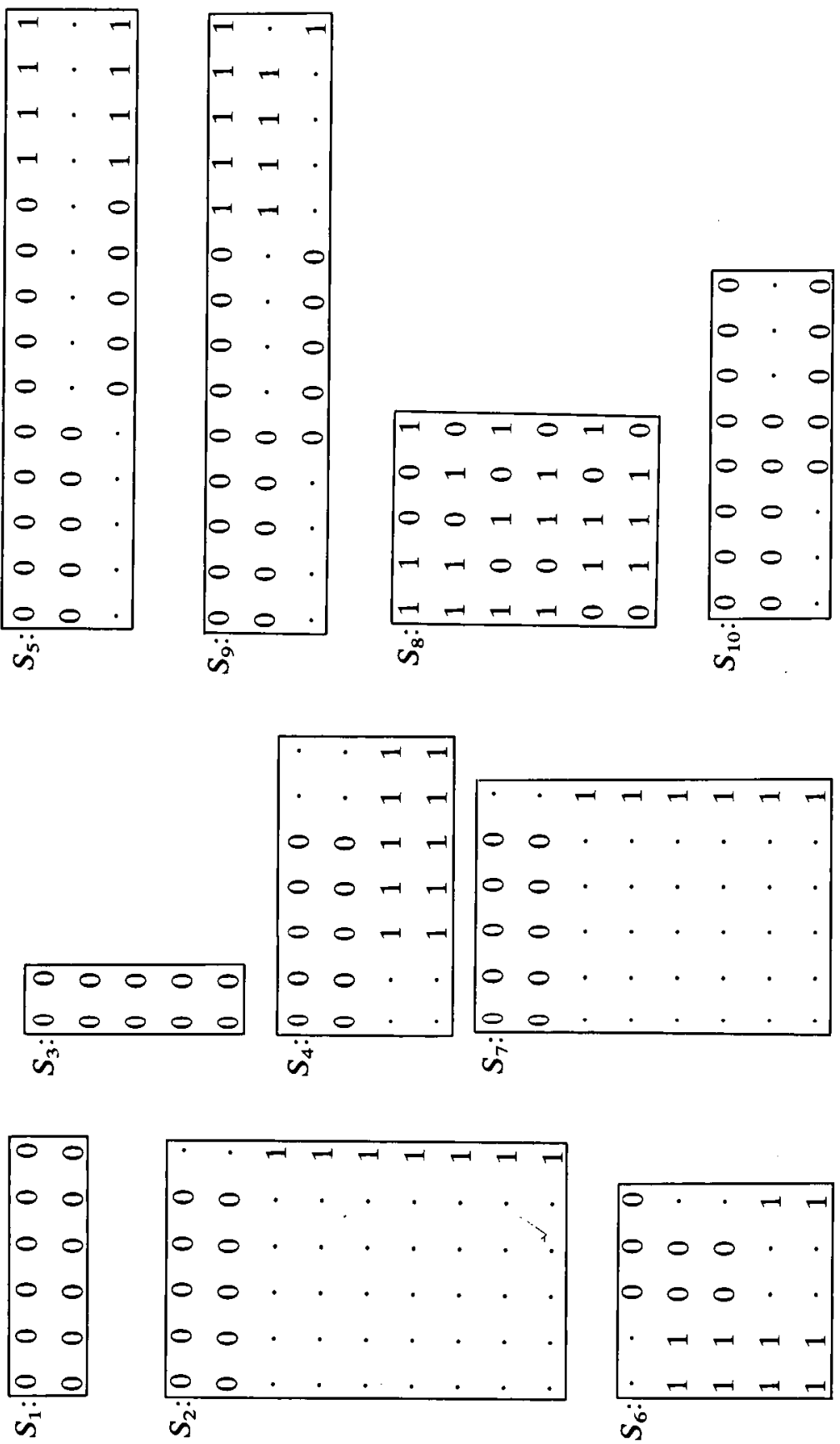
$(0 \leqq i \leqq 8)$ denote the number of columns of $M$ with exactly $i$ entries 0 . Then

yield

$$
\sum_{i=0}^{8} s_{i}=15, \quad \text { and } \quad \sum_{i=1}^{8} i s_{i} \geqq 72
$$

$$
s_{5}+2 s_{6}+3 s_{7}+4 s_{8} \geqq 12+4 s_{0}+3 s_{1}+2 s_{2}+s_{3} \geqq 12 .
$$

Together with (5) there are

$$
\sum_{i=3}^{8}\left(\begin{array}{l}
i \\
3
\end{array}\right) s_{i} \geqq 10\left(s_{5}+2 s_{6}+3 s_{7}+4 s_{8}\right) \geqq 120>2\left(\begin{array}{l}
8 \\
3
\end{array}\right)
$$

triples of entries 0 in the columns of $M$, so that $N \subset M$.

Lemma 5. If a column of $B$ has 9ientries 0 or 9 entries 1 , then $N$ or $\bar{N} \subset B$.

Proof. Let $b_{i, 1}=0$ for all $i$ with $1 \leqq i \leqq 9$. Rows 1 to 9 and columns 2 to 17 determine $M \subset B$. If there is a column of $M$ with at least 5 entries 0 , we use Lemma 4 . Otherwise at least $16\left(\begin{array}{l}5 \\ 2\end{array}\right)=160$ pairs with both entries 1 in the columns of $M$ distributed among all pairs of rows of $M$ guarantee 2 rows of $M$ having 5 columns with both entries 1 , since $4\left(\begin{array}{l}9 \\ 2\end{array}\right)<160$. Then $\bar{S}_{2} \subset B$, and we use Lemma 3 .

LemMa 6. If $S_{4}$ or $\bar{S}_{4} \subset B$, then $N$ or $\bar{N} \subset B$.

Proof. Let $S_{4}$ be in the first rows and columns of $B$. Rows 5 to 13 and columns 1 to 2 , 3 to 5,6 to 7 determine $M_{1}, M_{2}, M_{3}$, respectively. Either $-N_{1}$ or $\bar{N} \subset B$, or every row of $M_{2}$ has at most 2 entries 0 , and at most 2 entries 1 . In at least 5 rows of $M_{2}$ there are 2 entries 0 (1). Then either $N \subset B(\bar{N} \subset B)$, or $\bar{S}_{3} \subset M_{1}\left(S_{3} \subset M_{3}\right)$, and Lemma 4 can be used.

LEMMA 7. If $S_{5}$ or $\bar{S}_{5} \subset B$, then $N$ or $\bar{N} \subset B$.

Proof. Let $S_{5}$ occur in the first rows and columns of $B$. Rows 4 to 13 and columns 1 to 5 of $B$ determine $M_{1}$, and rows 4 to 13 together with columns 11 to 14 of $B$ determine $M_{2}$. At first $b_{3, j}=1$ for all $j$ with $1 \leqq j \leqq 5$, or Lemma 2 can be used. Next either $N \subset B$, or every row in $M_{1}$ has at least 3 entries 1 . If one row of $\dot{M}_{2}$ exists with more than one entry 1 , then together with row 3 of $B$ we have $S_{4} \subset B$, and use Lemma 6 . Otherwise every row of $M_{2}$ has 3 entries 0 , and any distribution of 10 triples 000 among the 4 columns of $M_{2}$ guarantees $N \subset M_{2}$, since $2\left(\begin{array}{l}4 \\ 3\end{array}\right)<10$.

LEMMA 8. $N$ or $\bar{N}$ exist in any $(5,5)$-matrix obtained by changing rows or columns of $S_{6}, S_{6}^{T}, \bar{S}_{6}$ or $\bar{S}_{6}^{T}$.

Proof. If the second or third element of column 5 of $S_{6}$ is 1 , then $\dot{\bar{N}} \subset S_{6}$, and otherwise $N \subset S_{6}$. 
LEMMA 9. If $S_{7} \subset B$ (respectively $\bar{S}_{7} \subset B$ ), then either $N$ or $\bar{N} \subset B$, or $S_{8}$ (respectively $\bar{S}_{8}$ ) are in rows 3 to 8 and columns 1 to 5 of $S_{7}$ (respectively $\bar{S}_{7}$ ), up to exchanges of rows or columns.

Proof. Let $S_{7} \subset B$, and $M$ denotes the elements of $S_{7}$ in rows 3 to 8 and columns 1 to 5. At least 3 entries 1 exist in every row of $M$, or $N \subset S_{7}$. If one row has more than 3 entries 1 , then $\left(\begin{array}{l}4 \\ 2\end{array}\right)+5\left(\begin{array}{l}3 \\ 2\end{array}\right)>2\left(\begin{array}{l}5 \\ 2\end{array}\right)$ implies that 2 columns exist in $M$ with 3 pairs 11 , and together with column 6 of $S_{7}$ it follows $\bar{N} \subset S_{7}$. It remains that $M$ has exactly 18 entries 1 . No column of $M$ has more than 4 entries 1 , since otherwise $\bar{S}_{3} \subset B$ (Lemma 4 ). No column of $M$ has less than 3 entries 1 , since otherwise 4 columns have 4 entries 1 , and this forces 2 rows of these 4 columns to have 2 entries 1 and 4 rows to have 3 entries 1 , and then $2\left(\begin{array}{l}2 \\ 2\end{array}\right)+4\left(\begin{array}{l}3 \\ 2\end{array}\right)>2\left(\begin{array}{l}4 \\ 2\end{array}\right)$ together with column 6 of $S_{7}$ guarantees $\bar{N} \subset S_{7}$. So $M$ can have only 3 columns with 4 , and 2 columns with 3 entries 1 . Then $N \subset S_{7}$ (with column 6), or by changing of rows or columns we obtain $M=S_{8}$.

LEMMA 10. If $S_{9}$ or $\bar{S}_{9} \subset B$, then $N$ or $\bar{N} \subset B$.

Proof. If we find $S_{9}$ in the first rows and columns of $B$, then by Lemma 2 we can assume $b_{3, j}=b_{2, j+5}=1$ for $j=1,2,3,4$. Let $M_{1}$ be determined by rows 4 to 13 and columns 1 to 9 . Either $N \subset B$, or in columns 1 to 5 , and in columns 5 to 9 of $M_{1}$, respectively, there are at least 30 entries 1 . Every column $\neq 5$ of $M_{1}$ has at most 6 entries 1 , or we can use Lemma 3. Thus column 5 must have at least 6 entries 1 . Since Lemma 5 yields $N$ or $\bar{N} \subset B$, column 5 has at most 8 entries 1 .

(i) $b_{i, 5}=1$ for all $i$ with $8 \leqq i \leqq 13, b_{i, 5}=0$ otherwise: The elements in rows 4 to 7 of $B$ and in columns 1 to 4,6 to 9,10 to 14 are denoted by $M_{2}, M_{3}, M_{4}$, respectively. Every row of $M_{2}$ and of $M_{3}$ has at least 3 entries 1 , otherwise $N \subset B$. Then every pair of rows of $M_{2}$ and of $M_{3}$ has at least 2 columns with both entries 1 . Together with rows 2 or 3 , and $M_{4}$, we find $N \subset B$, or every column of $M_{4}$ has at least 3 entries 0 . Then 2 columns of $M_{4}$ together with column 5 of $B$ yield $N \subset B$.

(ii) $b_{i, 5}=1$ for all $i$ with $7 \leqq i \leqq 13, b_{i, 5}=0$ otherwise: Let $M_{5}$ and $M_{6}$ denote the elements of $B$ in rows 4 to 6 , and in columns 1 to 4 , and 6 to 9 , respectively. Rows 7 to 13 and columns 1 to 4 determine $M_{7}$. Every row of $M_{5}$ and of $M_{6}$ has at least 3 entries 1 (otherwise $N \subset B$ ), and then at most 3 entries 1 (otherwise $\bar{N} \subset B$ ). Every column of $M_{5}$ and of $M_{6}$ has at most one entry 0 , otherwise $\bar{N} \subset B$. Thus we can assume $b_{4,1}=b_{5,2}=$ $b_{6,3}=b_{4,6}=b_{5,7}=b_{6,8}=0$, and 1 for all other elements of $M_{5}$ and of $M_{6}$. As in every pair of columns of $M_{7}$ at most 2 pairs 11 occur (otherwise together with column 5 of $B$ we obtain $\bar{N} \subset B$ ), there is a row in $M_{7}$ with 11 in columns 1 and 4,2 and 4 , or 3 and 4 , and we can assume $b_{7,3}=b_{7,4}=1$. At least 2 elements of $b_{7,6}$ to $b_{7,9}$ are 1 , otherwise $N \subset B$. These have to be $b_{7,6}$ and $b_{7,7}$, since rows $4,5,7$ and columns $3,4,8,9$ yield $\bar{N} \subset B$ or $b_{7,8}=b_{7,9}=0$. Then rows $5,6,7$ and columns $1,4,6$ imply $\bar{N} \subset B$ or $b_{7,1}=0$, and rows 4, 6, 7 and columns $2,4,7$ imply $\bar{N} \subset B$ or $b_{7,2}=0$. 
At least 14 entries 1 occur in $M_{7}$. Column 4 of $M_{7}$ contains at most 3 entries 1 , since otherwise we can use Lemma 3. No column of $M_{7}$ has more than 4 entries 1 , and thus two of columns 1 to 3 of $M_{7}$ contain exactly 4 entries 1 . After possibly changing columns 1 and 2 , and rows 4 and 5 we can assume $b_{8,1}=b_{9,1}=b_{10,1}=b_{11,1}=1$, and $b_{12,1}=b_{13,1}=0$. Now Lemma 9 guarantees $N$ or $\bar{N} \subset B$, or we can assume $S_{8}$ in rows $5,6,8,9,10,11$ and columns $6,9,5,7,8$, in these sequences.

Then rows 5,6 and $8,9,10$ or 11 and columns 1,4 and $6,6,9$ or 9 , respectively, yield $\bar{N} \subset B$ or $b_{8,4}=b_{9,4}=b_{10,4}=b_{11,4}=0$. Rows $4,5,10$ and columns $3,8,9$ yield $\bar{N} \subset B$ or $b_{10.3}=0$. Rows $4,6,11$ and columns $2,7,9$ yield $\bar{N} \subset B$ or $b_{11.2}=0$. Then rows $1,8,10$ and columns $3,4,7$, rows $1,7,9$ and columns $2,8,9$, rows $1,2,10$ and columns $2,3,4$, rows $1,2,11$ and columns $2,3,4$ yield $N \subset B$ or $b_{8,3}=b_{9,2}=b_{10.2}=b_{11.3}=1$, respectively. Furthermore $\bar{N} \subset B$ or $b_{8,2}=b_{9,3}=0$ follow from rows $8,9,10$ and columns 1,2 , 5, and from rows $8,9,11$ and columns $1,3,5$.

If $b_{12,3}=b_{13,3}=1$, then Lemma 4 can be used. Therefore we can assume $b_{12,3}=0$ after possibly changing rows 12 and 13 . Then rows $1,2,12$ and columns 1 to 4 imply $\underline{N} \subset B$ or $b_{12,2}=b_{12,4}=1$. Rows $4,6,12$ and columns $2,4,7$, and columns $2,4,9$ yield $\bar{N} \subset B$ or $b_{12,7}=b_{12,9}=0$. Then rows $1,3,12$ and columns 6 to 9 yield $N \subset B$ or $b_{12,6}=b_{12.8}=1$. It follows $b_{13,6}=0$, or Lemma 4 can be used. If $b_{13,2}=0$, then rows 1,2 , 13 and columns 1 to 4 yield $N \subset B$ or $b_{13,3}=b_{13,4}=1$, rows $1,7,13$ and columns $1,2,8$ yield $N \subset B$ or $b_{13,8}=1$, and then we find $\bar{N}$ in rows $4,5,13$ and columns $3,4,8$. If, however, $b_{13,2}=1$, then rows $10,12,13$ and columns $2,5,8$ yield $\bar{N} \subset B$ or $b_{13,8}=0$, rows $1,3,13$ and columns 6 to 9 yield $N \subset B$ or $b_{13.7}=b_{13,9}=1$, and then we find $\bar{N}$ in rows 4 , 6, 13 and columns $2,7,9$.

(iii) $b_{i .5}=1$ for all $i$ with $6 \leqq i \leqq 13, b_{i, 5}=0$ otherwise: At least 2 of the elements $b_{3,10}$ to $b_{3,13}$ of $B$ (say $b_{3,10}$ and $b_{3,11}$ ) are 0 , otherwise $\bar{N} \subset B$ (with rows 1 and 2). At least one of the elements $b_{4,10}, b_{4,11}, b_{5,10}, b_{5,11}$ (say $b_{4,10}$ ) is 1 , otherwise $N \subset B$ (with column 5). Columns 6 to 9 , rows 4 to 5 and rows 6 to 13 of $B$ determine $M_{8}$ and $M_{9}$, respectively. Both rows of $M_{8}$ have at least 3 entries 1 (otherwise $N \subset B$ ), and then at most 3 entries 1 (otherwise $\bar{N} \subset B$ ). If one column of $M_{8}$ has both entries 0 , then $\bar{N} \subset B$. Thus we can assume $b_{4.9}=b_{5.8}=0$, and all other elements of $M_{8}$ are 1 .

In every column of $M_{9}$ there are at most 4 entries 1 , or we can use Lemma 4 (with column 5). At least 30 entries 1 are in columns 5 to 9 of $M_{1}$, or $N \subset B$. Thus exactly 4 entries 1 occur in every column of $M_{9}$, and exactly 2 entries 1 in every row of $M_{9}$. We can assume $b_{6,9}=b_{7,9}=b_{8,9}=b_{9,9}=0$. If in 2 of rows 1 to 4 of $M_{9}$, and in columns 1 and 2 of $M_{9}$ there are 4 entries 1 , then we have found $\bar{S}_{3}$, and Lemma 4 can be used. Otherwise we can assume $b_{6,6}=b_{6,8}=b_{7,6}=b_{7,8}=1$, and $b_{6,7}=b_{7,7}=0$ (after possibly changing columns 6 and 7). Then in these sequences the elements of rows $3,6,7,2,4$ and of columns 6,8 , $7,9,10$ of $B$ represent $S_{6}$, and Lemma 8 completes the proof.

LEMMA 11. If $S_{10}$ or $\bar{S}_{10} \subset B$, then $N$ or $\bar{N} \subset B$.

Proof. Let $S_{10}$ be in the first rows and columns of $B$. Then $b_{2,6}=b_{2,7}=b_{2,8}=b_{3,1}=$ $b_{3,2}=b_{3,3}=1$, or $N \subset B$. The elements of $B$ in rows 4 to 13 and columns 1 to 3,4 to 5 , 
and 6 to 8 are denoted by $M_{1}, M_{2}$ and $M_{3}$, respectively. If we observe Lemma 4 , it suffices to discuss 3 cases: $M_{2}$ has (i) one row 00 , (ii) 4 rows 11 , or (iii) 4 rows 01 .

(i) $b_{4,4}=b_{4.5}=0$. Then $N \subset B$, or $b_{4,1}=b_{4,2}=b_{4,3}=b_{4,6}=b_{4,7}=b_{4,8}=1$. By Lemma 4 we can assume one entry 0 in each of rows 2 to 5 of $M_{2}$. The corresponding rows of $M_{1}$ and $M_{3}$ have exactly 2 entries 1 , since otherwise $N$ or $\bar{N} \subset B$. Then we can assume 2 equal rows in $M_{1}$ (say $b_{5,1}=b_{6,1}=b_{5,2}=b_{6,2}=1$ and $b_{5,3}=b_{6,3}=0$ ). As $M_{3}$ has at least one column with both entries 1 in rows 2 and 3 (say $b_{5,6}=b_{6,6}=1$ ), we find $\bar{N}$ in rows $4,5,6$ and columns $1,2,6$.

(ii) $b_{i, 4}=b_{i, 5}=1$ for all $i$ with $4 \leqq i \leqq 7$. In $M_{1}$ and $M_{3}$ rows 4 to 7 have at least one, and rows 8 to 13 at least 2 entries 1 (otherwise $N \subset B$ ). Together there are at least 16 entries 1 , which guarantee at least one column with 6 entries 1 in $M_{1}$, and in $M_{3}$, since by Lemma 3 we can assume at most 6 entries 1 in every column. At most 2 entries 1 exist in the first 4 rows of every column of $M_{1}$ and $M_{3}$ (otherwise $\bar{N} \subset B$ ). If a column with 6 entries 1 in $M_{1}$ or $M_{3}$ has exactly one entry 1 in the first 4 rows, then $N$ or $\bar{N} \subset B$ by Lemma 9 , since the existence of $S_{8}$ would force a row 11 in rows 8 to 13 of $M_{2}$, and then Lemma 4 can be used. As $M_{1}$ and $M_{3}$ cannot both have a column with 4 entries 0 in the first 4 rows (otherwise we use Lemma 4), we can assume that in $M_{1}$ a column exists, which has 6 entries 1 , and 2 of them in the first 4 rows. Thus we may choose $b_{i, 3}=1$ for $i=6,7, \ldots, 11$ and $b_{4.3}=b_{5.3}=b_{12.3}=b_{13.3}=0$. Then by Lemma 9 we can assume $S_{8}$ in rows 6 to 11 and columns 4 to 8 of $B$. After possibly changing rows 12 and 13 we have $b_{12,4}=b_{13,5}=1$, and $b_{12,5}=b_{13,4}=0$ (otherwise we have cases (i) or (iii), or $\bar{S}_{3}$, and Lemma 4 can be used). Together with rows 1 and 2 of $B$ we get $N \subset B$, or $b_{12,1}=b_{12,2}=b_{13,1}=$ $b_{13.2}=1$.

We next prove, that 6 entries 1 in columns 2 or 3 of $M_{3}$ yield $N$ or $\bar{N} \subset B$. After possibly changing rows 6 and 7,8 and 9,10 and 11 , so as columns 7 and 8 of $B$ we can assume $b_{12,8}=b_{13,8}=b_{5,8}=1$, and $b_{4,8}=0$ (rows 4 and 5 can be changed). By Lemma 9 we find $N$ or $\bar{N} \subset B$, or $S_{8}$ in rows $5,6,12,8,13,10$ and columns $4,5,1,3,2$ after possibly changing columns 1 and 2 of $B$. Thus we assume $b_{5,1}=b_{6,1}=b_{6,2}=b_{8,2}=b_{10,2}=0, b_{8,1}=$ $b_{10,1}=b_{5,2}=1$. Then rows $8,10,12,13$ and columns $1,6,8$ of $B$ yield $N \subset B$, or $b_{12,6}=b_{13,6}=0$. Rows 12 and 13 together with rows 1 and 3 of $B$ yield $N \subset B$, or $b_{12.7}=b_{13.7}=1$. Rows $8,9,10,11$ and columns $1,3,6$ of $B$ yield $\bar{N} \subset B$, or $b_{9,1}=b_{11,1}=0$. Together with rows 1 and 2 it follows $b_{9,2}=b_{11,2}=1$ (otherwise $N \subset B$ ). Rows 7, 9, 11 and columns $2,3,7$ yield $\bar{N} \subset B$, or $b_{7,2}=0$. Rows $1,6,7$ and columns $1,2,6$ force $N \subset B$, or $b_{7,1}=1$. Rows 5, 12, 13 and columns $2,7,8$ yield $\bar{N} \subset B$, or $b_{5,7}=0$. Rows $1,5,6$ and columns $1,6,7$ force $N \subset B$, or $b_{5,6}=1$. If now $b_{4,1}=1$, then 6 entries 1 are in column 1 of $M_{1}$, and by Lemma 9 it remains $b_{4,6}=1, b_{4,7}=0\left(S_{8}\right.$ is in rows $4,7,8,12,10,13$ and columns $4,5,8,7,6$ of $B$ ). Then in case $b_{4,2}=0$ we find $S_{3}$ in columns 2,7 and rows 1,4 , $6,8,10$ of $B$, and we use Lemma 4 , and in case $b_{4,2}=1$ rows $4,5,9$ and columns $2,4,6$ yield $\bar{N} \subset B$. If otherwise $b_{4,1}=0$, then rows $1,2,4$ and columns $1,2,3$ yield $N \subset B$, or $b_{4,2}=1$. Then $b_{4,7}=0$ forces $N \subset B$ (rows $1,4,5$ and columns $1,3,7$ ), and $b_{4,7}=1$ gives $\bar{S}_{3} \subset B$ (rows $4,9,11,12,13$, columns 2,7 ), and we use Lemma 4 .

In the following we can assume that at most 5 entries 1 exist in columns 2 and 3 of $M_{3}$, that is, 6 entries 1 occur in column 1 of $M_{3}$. By Lemma 9, this is possible only in two 
cases, either $b_{4,6}=b_{5,6}=1$ and $b_{12,6}=b_{13,6}=0$, or $b_{4,6}=b_{5,6}=0$ and $b_{12,6}=b_{13,6}=1$. In the first case we find $N$ in rows $1,3,12,13$ and columns 4 to 8 , or $b_{12,7}=b_{12,8}=b_{13,7}=b_{13.8}=$ 1. Then more than 5 entries 1 occur in column 2 or 3 of $M_{3}$, or $N \subset B$ (rows $1,4,5$, columns $3,7,8)$. In the second case we use Lemma 9, and we can assume $S_{8}$ in columns 3 , $2,1,4,5$ of $B$, and $(j)$ in rows $11,9,10,8,13,12$, or $(j j)$ in rows $11,8,10,9,13,12$ of $B$, after possibly changing columns 1 and 2 . In other words, we can assume $b_{10,2}=b_{11,1}=0$, $b_{10.1}=b_{11,2}=1$ and $(j) b_{8,2}=b_{9,1}=0, b_{8,1}=b_{9,2}=1$, or $(j j) b_{8,2}=b_{9,1}=1, b_{8,1}=b_{9,2}=0$.

In case $(j)$ rows $9,11,12,13$ and columns $2,6,7$ yield $\bar{N} \subset B$ or $b_{12,7}=b_{13,7}=0$, rows $8,10,12,13$ and columns $1,6,8$ yield $\bar{N} \subset B$ or $b_{12,8}=b_{13,8}=0$, and then we have $N$ in rows $1,12,13$ and columns $3,7,8$.

In case $(j j) b_{4,7}=b_{5,7}=0$ or 1 yield $N$ in rows $1,4,5$ and columns $3,6,7$, or $\bar{N}$ in rows $4,5,7$ and columns $4,5,7$, respectively, and thus we can assume $b_{4,7}=0, b_{5,7}=1$, after possibly changing rows 4 and 5 . Then rows $1,3,4$ and columns $6,7,8$ imply $N \subset B$ or $b_{4,8}=1$, and rows $4,5,6$ and columns $4,5,8$ imply $\bar{N} \subset B$ or $b_{5,8}=0$. If $b_{4,2}=0$, then $b_{5,2}=1$ or $N$ is in rows $1,4,5$ and columns $2,3,6$. Rows $5,11,13$ and columns $2,5,7$ yield $\bar{N} \subset B$ or $b_{13,7}=0$. Then rows $1,3,13$ and columns $4,7,8$ imply $N \subset B$ or $b_{13,8}=1$. Rows $4,10,13$ and columns $1,5,8$ then yield $\bar{N} \subset B$ or $b_{4,1}=0$, and we find $N$ in rows 1 , 2,4 and columns $1,2,3$. If, otherwise, $b_{4,2}=1$, then $b_{6,2}=0$ or $\bar{N}$ is in rows $4,6,8$ and columns $2,4,8$, and $b_{12,8}=0$ or $\bar{N}$ is in rows $4,8,12$ and columns $2,4,8$. Rows $1,3,12$ and columns $5,7,8$ yield $N \subset B$ or $b_{12,7}=1$. Then rows $5,9,12$ and columns $1,4,7$ yield $\bar{N} \subset B$ or $b_{5,1}=0$. Rows $11,12,13$ and columns $2,6,7$ yield $\bar{N} \subset B$ or $b_{13,7}=0$. Then rows $1,3,13$ and columns $4,7,8$ imply $N \subset B$ or $b_{13,8}=1$. At last rows $4,10,13$ and columns $1,5,8$ yield $\bar{N} \subset B$ or $b_{4,1}=0$, and we find $N$ in rows $1,4,5$ and columns $1,3,6$.

(iii) $b_{i, 4}=0, b_{i, 5}=1$ for all $i$ with $4 \leqq i \leqq 7$. There are at most 13 entries 1 in $M_{2}$ (otherwise (ii)). At least 3 entries 1 are in every row of rows 4 to 13 in columns 1 to 5 , or in 4 to 8 (otherwise $N \subset B$ ). Thus at least 17 entries 1 exist in $M_{1}$, and in $M_{3}$, and either we use Lemma 3 , or at least 2 columns of $M_{1}$, and at least 2 columns of $M_{3}$ have exactly 6 entries 1.

Every column in the first 4 rows of $M_{1}$ or of $M_{3}$ has at least 2 entries 1 (otherwise $S_{3} \subset B$, and we use Lemma 4). In the first 4 rows of $M_{1}$ and $M_{3}$ there exists at most one column with 4 entries 1 (otherwise $\bar{N} \subset B$ ), which we can assume not to be in $M_{1}$. Each of the first 4 rows of $M_{1}$ and of $M_{3}$ has at least 2 entries 1 (otherwise $N \subset B$ ). Altogether the first 4 rows of $M_{1}$ contain at least 8 entries 1 , so that 2 columns have exactly 3 entries 1 , and we can assume $b_{4,3}=b_{5,3}=b_{6,3}=b_{4,2}=b_{5,2}=b_{7,2}=1, b_{6,2}=b_{7,3}=0$ (otherwise $\bar{N} \subset B$ ). Then $b_{6,1}=b_{7,1}=1$, or $N \subset B$.

After possibly changing rows 6 and 7 , and columns 2 and 3 of $B$ we can assume column 3 to be one of the 2 columns of $M_{1}$ with 6 entries 1 , that is, $b_{8,3}=b_{9,3}=b_{10,3}=1$. Then Lemma 9 can be used, and we find $S_{8}$ in rows $4,8,5,9,6,10$ and columns $8,7,6$, 4,5 of $B$ (columns 4 and 5 of $S_{8}$ have to be in $M_{2}$ ), that is,

$$
\begin{aligned}
b_{4,7}=b_{4,8}=b_{5,6}=b_{5,8}=b_{6,6}=b_{6,7}=b_{8,4}=b_{8,7} & =b_{8,8} \\
& =b_{9,4}=b_{9,6}=b_{9,8}=b_{10,4}=b_{10,6}=b_{10,7}=1,
\end{aligned}
$$


and

$$
b_{4,6}=b_{5,7}=b_{6,8}=b_{8,5}=b_{8,6}=b_{9,5}=b_{9,7}=b_{10,5}=b_{10,8}=0 .
$$

In rows $4,5,7$ and columns $2,5,8$ of $B$ we find $\bar{N}$, or $b_{7,8}=0$, and then $b_{7,6}=b_{7,7}=1$ (otherwise $N \subset B$ ). Rows $4,5,8,9$ and columns $2,3,8$ of $B$ yield $\bar{N} \subset B$, or $b_{8,2}=b_{9,2}=0$.

If 6 entries 1 are in column 1 of $M_{1}$, then $N$ or $\bar{N} \subset B$ by Lemma $9\left(S_{8}\right.$ is impossible, since rows 6 and 7 are identical in columns 4 to 8 ). Thus 6 entries 1 exist in column 2 of $M_{1}$. Then Lemma 9 can be used, and columns 4 and 5 of $S_{8}$ have to be in $M_{2}$. Then at most 11 entries 1 exist in $M_{2}$, and therefore $M_{1}$ has more than 18 entries 1 , that means, at least one column of $M_{1}$ has 7 entries 1 , and Lemma 3 can be used.

Lemma 12. Let $Z_{i}$ denote the number of pairs of rows of $B$ with $i$ equal columns. Then either $N$ or $\bar{N} \subset B$, or

$$
Z_{9}+Z_{10} \geqq 12+\sum_{i \leq 6} Z_{i}
$$

Proof. If $Z_{i}>0$ for $i>10$, then by Lemma 2 we have $N$ or $\bar{N} \subset B$. Otherwise, if $s_{i}$ denotes the number of columns with $i$ entries 0 , then

$$
\begin{gathered}
\sum_{i \leq 10} Z_{i}=\left(\begin{array}{c}
13 \\
2
\end{array}\right)=78, \text { and } \sum_{i \geq 0} s_{i}=17, \\
\sum_{i \leq 10} i Z_{i}=\sum_{i \geq 0}\left(\left(\begin{array}{c}
i \\
2
\end{array}\right)+\left(\begin{array}{c}
13-i \\
2
\end{array}\right)\right) s_{i} \geqq 36 \sum_{i \geq 0} s_{i}=612 .
\end{gathered}
$$

At most 2 pairs of rows in every triple of rows of $B$ have an even number of equal columns (Lemma 1, $c=17$ ). Again the Theorem of Turán ([2], p. 17) implies then

$$
Z_{0}+Z_{2}+Z_{4}+Z_{6}+Z_{8}+Z_{10} \leqq\left[13^{2} / 4\right]=42 \text {. }
$$

With (7) and $Z_{8}+Z_{10}$ from (9) we obtain

$$
\begin{aligned}
\sum_{i \leq 10} i Z_{i} & =2\left(Z_{9}+Z_{10}\right)+Z_{8}+Z_{10}+7 \sum_{i \leq 10} Z_{i}-\sum_{i \leq 6}(7-i) Z_{i} \\
& \leqq 2\left(Z_{9}+Z_{10}\right)+588-2 \sum_{i \leq 6} Z_{i} .
\end{aligned}
$$

From (8) and (10) we obtain (6).

LEMMA 13. If there are three rows $a, b, c$ in $B$, so that the number of equal columns is at least 9 in rows $a$ and $b$, and in rows $a$ and $c$, then $N$ or $\bar{N} \subset B$.

Proof. Let $a, b, c$ be rows $1,2,3$ of $B$. If more than 5 columns with both entries 0 (or both entries 1 ) occur in one pair of rows we use Lemma 2 . If then the pairs of rows 1 and 2 , and of rows 1 and 3 both contain 5 columns with both entries 0 , or both contain 5 columns with both entries 1 , then $N$ or $\bar{N} \subset B$ follows directly, or by use of Lemmas 7,10 , or 11 . Thus in the following we can assume 5 pairs with both entries 0 in the first 5 columns of rows 1 and 2, 5 pairs with both entries 1 in columns 6 to 10 of rows 1 and 3, and exactly 4 
columns with both entries 1 in rows 1 and 2 , and exactly 4 columns with both entries 0 in rows 1 and 3 .

If in rows 2 and 3 there are less than 7 equal columns, then by Lemma 12 we have $Z_{9}+Z_{10} \geqq 13$ in $B$. Then the pigeonhole principle guarantess that one row of $B$ exists which has together with each of two other rows at least 9 equal columns, among which in both pairs of rows occur 5 columns with both entries 0 , or 5 columns with both entries 1 . Thus we can use Lemmas 7,10 or 11 to get $N$ or $\bar{N} \subset B$.

In columns 11 to 17 occur at least 2 columns with both entries 0 in rows 1 and 3, and at least 2 columns with both entries 1 in rows 1 and 2 . We can assume $b_{1,11}=b_{1,12}=$ $b_{3,11}=b_{3,12}=0$ and $b_{1,13}=b_{1,14}=b_{2,13}=b_{2,14}=1$. Then $S_{1}$ or $\bar{S}_{1} \subset B$, or $b_{2,11}=b_{2,12}=1$ and $b_{3,13}=b_{3,14}=0$. Now at least 7 equal columns in rows 2 and 3 are possible only if at least 4 of them occur in columns 1 to 10 . More than 4 columns, however, yield $N$ or $\bar{N} \subset B$. Thus we can choose $b_{3,1}=b_{3,2}=b_{2,8}=b_{2,9}=b_{2,10}=0, b_{3,3}=b_{3,4}=b_{3.5}=b_{2,6}=b_{2,7}=$ 1 , and without loss of generality $b_{2,15}=b_{2,16}=b_{3,15}=b_{3,16}=1$. Then $\bar{N} \subset B$, or $b_{1,15}=$ $b_{1,16}=0$.

If column 15 in rows 4 to 13 contains more than 6 entries 0 , or more than 5 entries 1 , then $S_{2}$ or $\bar{S}_{2} \subset B$, and we can use Lemma 3. It remains to consider that column 15 in rows 4 to 13 contains (i) 5 entries 1 , and (ii) 6 entries 0 .

(i) $b_{i, 15}=1$ for all $i$ with $4 \leqq i \leqq 8$. By Lemma 9 we can assume $S_{8}$ in rows 3 to 8 and columns 1 to 5 . There exist 3 rows in this $S_{8}$ which together with row 3 of $B$ have 2 columns with both entries 1 . If one of these rows contains more than one entry 1 in columns 6 to 10 , then $\bar{S}_{10} \subset B$, and we use Lemma 11. Otherwise in columns 6 to 10 we find $\bar{N}$, or we have 3 rows with at least 4 entries 0 , and 8 rows with at least 3 entries 0 . Then any distribution of $3\left(\begin{array}{l}4 \\ 2\end{array}\right)+8\left(\begin{array}{l}3 \\ 2\end{array}\right)=42$ pairs 00 among the columns 6 to 10 guarantees $S_{3} \subset B\left(\right.$ since $\left.4\left(\begin{array}{l}5 \\ 2\end{array}\right)<42\right)$, and we use Lemma 4 .

(ii) $b_{i, 15}=0$ for all $i$ with $4 \leqq i \leqq 9$. By Lemma 9 we can assume $\bar{S}_{8}$ in rows 4 to 9 and columns 6 to 10 . If column 16 has at least 4 entries 0 in rows 4 to 9 , then $S_{3} \subset B$ (together with row 1 of $B$ ), and Lemma 4 can be used. Thus we can assume $b_{4,16}=b_{5,16}=b_{6,16}=1$. If then in columns 3 to 5 in one of rows 4 to 6 there are 2 entries 1 , then $\bar{S}_{10} \subset B$, and we use Lemma 11. Otherwise $N \subset B$, or in rows 4 to 6 and columns 1 and 2 occur entries 1 only. Then, however, we find $\bar{N}$ in rows 4 to 6 and columns 1,2 and 16, and Lemma 13 is proved.

The proof of Theorem 2 is complete, since Lemma 12 for any $B$ guarantees the existence of three rows which enable us to apply Lemma 13.

\section{REFERENCES}

1. R. K. Guy, (Ed.), Sixth British Combinatorial Conference, Unsolved Problems, No. 13 (Typescript 1977).

2. F. Harary, Graph theory, (Addison-Wesley, 1969). 
3. H. Harborth and I. Mengersen, Ein Extremalproblem für Matrizen aus Nullen und Einsen, J. Reine Angew. Math. 309 (1979), 149-155.

4. R. W. Irving, A bipartite Ramsey problem and the Zarankiewicz numbers. Glasgow Math. J. 19 (1978), 13-26.

TeChNisChe Universität BraunschweIG

D-3300 BraunschWEIG,

West Germany 